\title{
PANCREAS
}

\section{Characterisation of oestrogen receptor, progesterone receptor, trefoil factor 1 , and epidermal growth factor and its receptor in pancreatic cystic neoplasms and pancreatic ductal adenocarcinoma}

\author{
T-S Yeh, Y-Y Jan, C-T Chiu, Y-B Ho, T-C Chen, K-F Lee, K-M Chan, J-C Hsu,T-L Hwang, \\ M-F Chen
}

Gut 2002;51:712-716

Background and aims: The pancreatic cystic neoplasms, including solid pseudopapillary tumour (SPT), mucinous cystic neoplasm (MCN), and intraductal papillary mucin producing tumour (IPMT), have their characteristic clinicopathological features. A systematic investigation of oestrogen receptor $(O R)$, progesterone receptor (PR), trefoil factor $1(T F F 1)$, and epidermal growth factor and its receptor (EGF and EGFR) expressed in pancreatic cystic neoplasms and pancreatic ductal adenocarcinoma was determined to elucidate their corresponding sex and age predilection, cell origin, and pathway of malignant transformation.

Methods: Surgical specimens of SPT ( $n=10), M C N(n=12)$, IPMT $(n=10)$, and ductal adenocarcinoma $(n=20)$ were studied. The expression of OR, PR, TFF 1, EGF, and EGFR were each determined in each disease entity using monoclonal antibodies by immunohistochemical method. The results were correlated with the clinicopathological data.

Results: PR was expressed in all 10 SPT, whereas OR was expressed in none of 10 SPT. TFF 1 was not or weakly expressed in SPT. Although EGF was strongly expressed in seven of 10 SPT, synchronous expression of EGF and its receptor was expressed in none of 10 SPT. Of the $12 \mathrm{MCN}$, six had PR expression in the stroma cells but not in the neoplastic epithelium, seven had a moderate or strong expression of TFF 1, and 10 had no or weak EGFR expression, irrespective of their benigneity or malignancy. Synchronous expression of EGF and EGFR was observed in only one of 12 MCN. Among 10 IPMT, TFF 1 and EGFR were moderately or strongly expressed in all six malignancies, whereas TFF 1 and EGFR were not or weakly expressed in three of four benigneity. Of 20 ductal adenocarcinomas, TFF1 and EGFR were moderately or strongly expressed in 16 and 12, respectively. Synchronous expression of EGF and EGFR was observed in six of 10 IPMT and nine of 20 ductal adenocarcinoma, respectively.

Conclusion: PR was uniquely expressed in SPT, and OR and PR were expressed in stroma of MCN,

See end of article for uthors' affiliations

Correspondence to: Dr T- S Yeh, Dpartment of Surgery, Chang Gung Memorial Hospital, 5 Fu-Hsing Street, Taoyuan, Taiwan;

tsy471027@adm.cgmh.org.tw

Accepted for publication 30 April 2002 reflecting their sex and age predilection. TFFl expression was related to EGFR such as in IPMT and ductal adenocarcinoma, not related to EGFR such as in MCN, and not related to hormonal receptors such as in SPT. EGF and its receptor might play a part in the malignant transformation of IPMT and ductal adenocarcinoma, but not of SPT and MCN.
C ystic neoplasms of the pancreas are uncommon tumours that comprise $10 \%$ to $15 \%$ of all pancreatic cystic masses and only $1 \%$ of pancreatic malignancies. ${ }^{1}$ In 1978, Compagno and Oertel described a histopathological classification of cystic neoplasms of the pancreas, mentioning two different types with extremely different biological behaviour. $^{2}$ The serous cystadenoma was nearly almost benign, whereas the mucinous cystic neoplasm (MCN) with overt or latent malignancy, was thought to be capable of progression to invasive mucinous cystadenocarcinoma. In 1986, a subtype of mucinous cystadenoma was first described in five Japanese patients. ${ }^{3}$ This variant was called ductectatic cystic neoplasm. Since 1996, based on WHO nomenclature, the formerly mucinous ductal ectasia has been separated from MCN and assigned as intraductal papillary mucin producing tumour of the pancreas (IPMT). ${ }^{4}$ IPMT shares some common characteristics with MCN, such as its rare incidence, mucin production, cystic mass, less invasiveness, and better prognosis after resection compared with those of pancreatic ductal adenocarcinoma. ${ }^{5-10}$ However, there are unique clinicopathological features for each type. Some $60 \%$ to $80 \%$ of IPMT occur in the head of the pancreas and are characterised by cystic dilatation of the pancreatic duct attributable to a large amount of mucin secreted through a patulous orifice of the papilla of Vater, usually manifesting as acute pancreatitis. ${ }^{11}$ The peak age of IPMT patients of both sexes is the sixth decade. MCN usually presents with large unilocular or multilocular cysts in the body and tail of the pancreas of middle aged women. Solid pseudopapillary tumour of the pancreas (SPT) is another specified disease entity that is supposed to originate from pleuripotential embryonal stem cells rather than from acinar cells. ${ }^{12-14}$ This tumour is found strikingly in young female patients and is considered a low grade malignancy. Its prognosis after surgical excision is excellent. Nevertheless, aggressive behaviour, with an extension of the tumour into surrounding organs or vessels, local recurrence, or distant metastasis, has been sporadically reported. ${ }^{15}$

Abbreviations: $\mathrm{OR}$, oestrogen receptor; $\mathrm{PR}$, progesterone receptor; TFF 1, trefoil factor 1; EGF, epidermal growth factor; EGFR, epidermal growth factor receptor; SPT, solid pseudopapillary tumour of the pancreas; MCN, mucinous cystic neoplasm of the pancreas; IPMT, intraductal papillary mucin producing tumour of the pancreas; PBS, phosphate buffered saline 

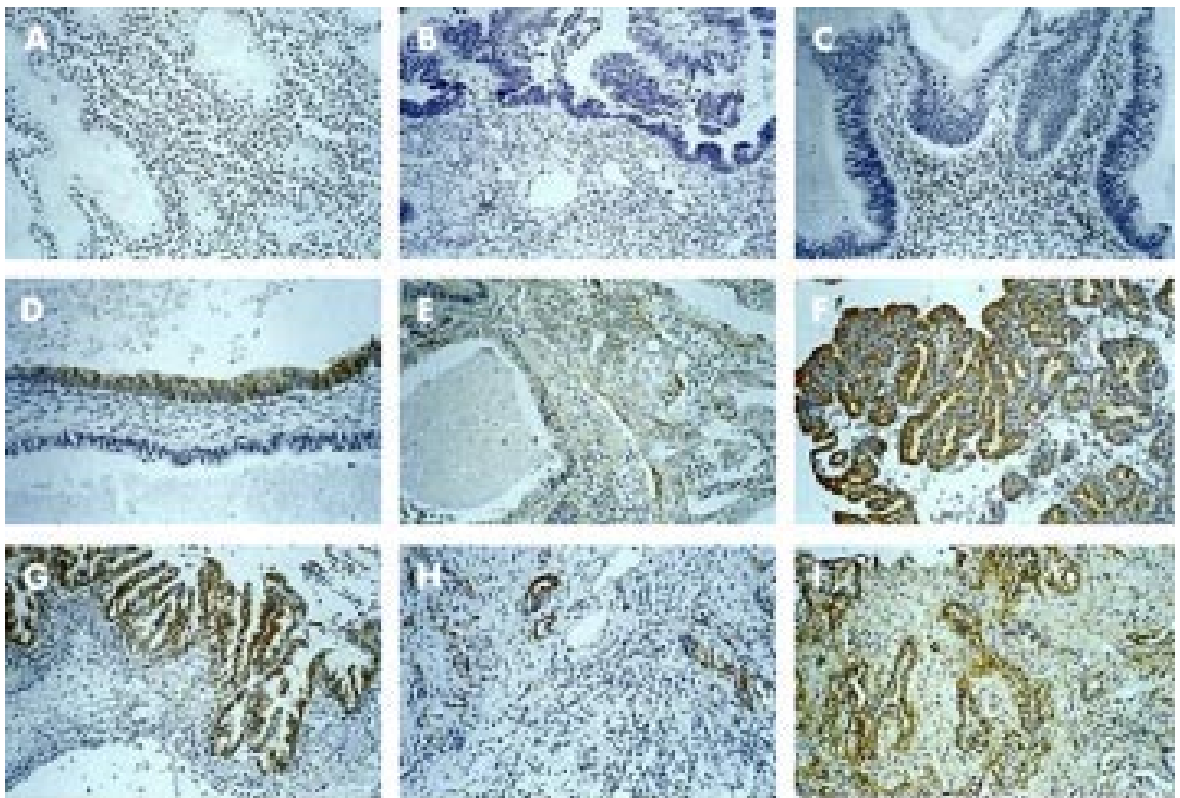

Figure 1 (A) Progesterone receptors (PR) were extensively expressed in the nuclei of cells of solid pseudo papillary tumours of the pancreas. (B) Oestrogen receptors were expressed in the nuclei of the stroma cells of mucinous cystic neoplasm (MCN) but not in the neoplastic epithelium. (C) PR were expressed in the nuclei of the stroma cells of MCN. (D) TFF1 were moderately expressed in the neoplasic epithelium of $M C N$. (E) Epidermal growth factor receptors (EGFR) were weakly expressed in MCN. (F) TFF1 were strongly expressed in intraductal papillary mucin producing tumour of the pancreas (IPMT). (G) EGFR were strongly expressed in IPMT. (H) TFF1 were moderately expressed in pancreatic ductal adenocarcinoma. (I) EGFR were strongly expressed in pancreatic ductal adenocarcinoma. (All panels original magnification $\times 100$ ).
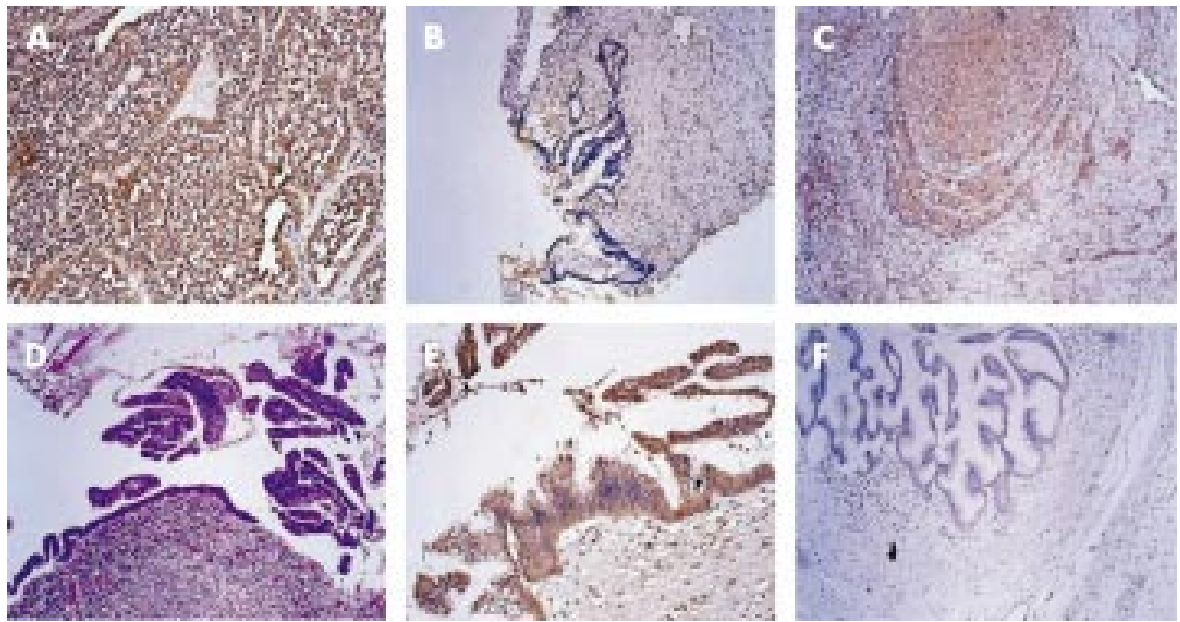

Figure 2 (A) Epidermal growth factor (EGF) were strongly expressed in solid pseudopapillary tumour of the pancreas (original magnification $\times 200$ ). (B) EGF were not expressed in mucinous cystic neoplasm (original magnification $\times 100$ ). (C) EGF were strongly expressed in pancreatic ductal adenocarcinoma. In this case, cancerous perineural invasion was noted, and EGF were expressed in the involved nerve fibres too (original magnification $\times 100$ ). (D) Intraductal papillary mucin producing tumour of the pancreas (IPMT) with severe dysplasia histologically (haematoxylin and eosin stain, original magnification $\times 100$ ). (E) EGF were strongly expressed in IPMT (the same as with $D$, original magnification $\times 200$ ). (F) Epidermal growth factor receptor were moderately expressed in IPMT (the same as with $D$, original magnification $\times 100)$.

Based on the unique characteristic clinicopathological features of the each disease entity aforementioned, we systematically determined the expression of oestrogen receptor (OR), progesterone receptor (PR), trefoil factor l (TFFl), and epidermal growth factor and its receptor (EGF and EGFR) in pancreatic cystic neoplasms and ductal adenocarcinoma, which might elucidate their corresponding sex and age predilection, cell origin, and pathway of malignant transformation.

\section{METHODS}

\section{Patients}

Patients with SPT $(n=10), \operatorname{MCN}(n=12)$, IPMT $(n=10)$, and pancreatic ductal adenocarcinoma $(n=20)$ who had undergone curative resection from January 1993 to December 1998 in Chang Gung Memorial Hospital were recruited for this study. All 10 patients with SPT were women with a mean age of 34 years (range, 18 to 47 years). The mean size of the 10 SPT was $7.8 \mathrm{~cm}$ in maximal dimension (range, 6 to $10.5 \mathrm{~cm}$ ). Four patients underwent Whipple's operation, whereas the remaining six patients underwent distal pancreatectomy. Among the 12 patients with MCN, there were five men and seven women with a mean age of 45 years (range, 19 to 70 years). Five patients underwent Whipple's operation, and the remaining seven patients underwent distal pancreatectomy. For those 10 patients with IPMT, there were six men and four women, with a mean age of 68 years (range, 54 to 76 years). Whipple's operation, 
Table 1 Expression of OR, PR, TFF1, and EGFR in pancreatic cystic neoplasms and pancreatic ductal adenocarcinoma

\begin{tabular}{|c|c|c|c|c|c|c|c|c|c|c|}
\hline & \multirow[b]{2}{*}{ OR } & \multirow[b]{2}{*}{ PR } & \multicolumn{4}{|l|}{ TFF1 } & \multicolumn{4}{|l|}{ EGFR } \\
\hline & & & +++ & ++ & + & - & +++ & ++ & + & - \\
\hline \multicolumn{11}{|l|}{ SPT $(n=10)$} \\
\hline Benign $(n=8)$ & 0 & 8 & 0 & 0 & 2 & 6 & 0 & 0 & 6 & 2 \\
\hline Malignant $(n=2)$ & 0 & 2 & 0 & 0 & 0 & 2 & 0 & 0 & 2 & 0 \\
\hline \multicolumn{11}{|l|}{$M C N(n=12)$} \\
\hline Benign $(n=4)$ & 2 & 4 & 0 & 2 & 2 & 0 & 0 & 1 & 2 & 1 \\
\hline Malignant $(n=8)$ & 2 & 2 & 1 & 4 & 3 & 0 & 0 & 1 & 3 & 4 \\
\hline \multicolumn{11}{|l|}{ IPMT $(n=10)$} \\
\hline Benign $(n=4)$ & 0 & 0 & 0 & 1 & 2 & 1 & 0 & 1 & 1 & 2 \\
\hline Malignant $(n=6)$ & 0 & 0 & 4 & 2 & 0 & 0 & 3 & 3 & 0 & 0 \\
\hline Ductal carcinoma $(n=20)$ & 0 & 0 & 7 & 9 & 3 & 1 & 4 & 8 & 5 & 3 \\
\hline
\end{tabular}

SPT, solid pseudopapillary tumour of pancreas; MCN, mucinous cystic neoplasm; IPMT, intraductal papillary mucin producing tumor; OR, oestrogen receptor; PR, progesterone receptor; EGFR, epidermal growth factor receptor.,,,++++++- represent strongly, moderately, weakly positive, and negative, respectively.

Table 2 EGF and its receptor immunoreactivity in pancreatic cystic neoplasms and pancreatic ductal adenocarcinoma

\begin{tabular}{llll}
\hline & \multicolumn{3}{l}{$\begin{array}{l}\text { Number of cases with } \\
\text { immunoreactivity to }\end{array}$} \\
\cline { 2 - 4 } Disease entity & EGF & EGFR & EGF + EGFR \\
\hline $\begin{array}{l}\text { SPT } \\
\text { Benign }(n=8)\end{array}$ & 6 & 0 & 0 \\
$\begin{array}{l}\text { Malignant }(n=2) \\
\text { MCN }\end{array}$ & 1 & 0 & 0 \\
$\quad$ Benign $(n=4)$ & 0 & 1 & 0 \\
Malignant $(n=8)$ & 2 & 1 & 1 \\
$\begin{array}{l}\text { PMT } \\
\text { Benign }(n=4)\end{array}$ & 3 & 1 & 1 \\
$\quad \begin{array}{l}\text { Malignant }(n=6) \\
\text { Ductal carcinoma }(n=20)\end{array}$ & 5 & 6 & 5 \\
\hline
\end{tabular}

Abbreviations as in table 1 . The scale of immunoreactivity +++ and ++ are regarded as immunopositivity, whereas + and - are regarded as immunonegativity (see Methods).

nearly total pancreatectomy, and distal pancreatectomy were performed in five, two, and three patients, respectively. Among the 20 patients with pancreatic ductal carcinoma, there were 14 men and six women with a mean age of 58 years (range, 39 to 72 years). Eighteen of the 20 patients underwent Whipple's operation and the remaining two underwent distal pancreatectomy.

\section{Immunohistochemical stainings for OR, PR, TFF1, EGF, and EGFR}

Formalin fixed, paraffin wax embedded tissues were cut into $4 \mu \mathrm{m}$ sections and mounted on glu coated slides. A modification of the avidin-biotin-peroxidase complex immunohistochemical method was performed. Slides were heated at $60^{\circ} \mathrm{C}$ for 60 minutes, then deparaffinised in xylene, and rehydrated in graded alcohols. Endogenous peroxidase was blocked by incubation in $0.3 \%$ hydrogen peroxidase in methanol, and slides were rehydrated and washed in phosphate buffered saline (PBS), pH 7.4 for 15 minutes. Sections were then blocked with $10 \%$ normal goat serum in PBS with $1 \%$ bovine serum albumin for 15 minutes. The blocking serum was decanted and various primary antibodies (OR, 1:50, monoclonal, mouse, antihuman, clone ID5, DAKO; PR, 1:50, monoclonal, mouse, antihuman, clone pgR636, DAKO; TFF1, 1:50, monoclonal, mouse, antihuman, clone HIl, DAKO; EGF-R, 1:200, monoclonal, mouse, antihuman, clone BC04, DAKO; and EGF, $0.1 \mathrm{mg} / \mathrm{ml}, \mathrm{DAKO}$ ) were applied, respectively, in PBS with $1 \%$ bovine serum albumin for 16 hours at $4^{\circ} \mathrm{C}$. Slides were washed in PBS with $1 \%$ Tween 20 for 10 minutes. After three PBS rinses, biotinylated goat antirabbit immunoglobulin
(Vector Labs, Burlingame, CA) at a dilution of $1 / 500$ was applied for 30 minutes at room temperature. After another PBS rinse, avidin DH: biotinylated horseradish peroxidase complex (Vector Labs) was applied for 30 minutes. After a final PBS rinse, the tissue sections were reacted with $0.06 \%$ diaminobenzidine (Sigma Chemical) for five minutes, rinsed, counterstained with haematoxylin, dehydrated with graded alcohols, cleared xylene, and coverslipped with permount.

The interpretation of OR and PR reactivity was performed as either negative or positive according to the criteria of Reiner et al. ${ }^{16}$ Quantification of TFF1, EGF, and EGFR was performed using a scale of - (negative), + (weakly positive), ++ (moderately positive), and +++ (strongly positive), taking into account the percentage of stained cells and the intensity of staining.

\section{RESULTS}

The expression of OR, PR, TFF1, and EGFR in SPT $(n=10)$, MCN $(n=12)$, IPMT $(n=10)$, and ductal adenocarcinoma $(n=20)$, respectively, were tabulated (table 1$)$. OR was expressed in none of 10 SPT, whereas PR was unexceptionally expressed in all 10 SPT (fig lA). TFFl and EGFR were not or weakly expressed in SPT. Of the $12 \mathrm{MCN}$, four had OR expression (fig lB) and six had PR expression (fig lC) in the nuclei of the stroma cells rather than in their neoplastic epithelium. All these six patients with a PR expression in the stroma cells were women. Seven had a moderate or strong expression of TFFl (fig 1D), whereas 10 had no or weak EGFR expression, irrespective of their benigneity or malignancy (fig $1 \mathrm{E}$ ). Of 10 IPMT, TFFl and EGFR were moderately or strongly expressed in six malignancies (fig $1 F$ and fig $1 G$ ), whereas TFFl and EGFR were not or weakly expressed in three of four benigneity. Of the 20 ductal adenocarcinomas, OR and PR were expressed in none, whereas TFFl and EGFR were moderately or strongly expressed in 16 and 12 , respectively (fig $1 \mathrm{H}$ and fig II). Furthermore, the expression of EGF associated with its receptor in SPT, MCN, IPMT, and ductal adenocarcinoma was shown (fig 2 and table 2). In brief, although EGF was strongly expressed in seven of 10 SPT, synchronous expression of EGF and its receptor was observed in none of 10 SPT. Synchronous expression of EGF and its receptor was observed in only one of eight malignant MCN. Synchronous expression of EGF and its receptor was observed in five of six malignant IPMT, and in one of four benign IPMT, which was considered severe dysplasia histologically, respectively. Synchronous expression of EGF and its receptor was observed in nine of 20 ductal adenocarcinomas.

\section{DISCUSSION}

EGF has a growth promoting or trophic effect on many tissues, including the pancreas. ${ }^{17}{ }^{18}$ An overexpression of EGFR has 
been identified in pancreatic ductal adenocarcinoma, along with increased amounts of EGF, which suggests the establishment of an autocrine loop. ${ }^{19}$ In this study, EGFR represented as an important tumourigenic factor in the group of IPMT and ductal adenocarcinoma. All six histologically malignant IPMT had a moderate or strong EGFR expression, whereas three of four benign IPMT had either no or weak EGFR expression. Of significance, one benign IPMT with synchronous expression of EGF and its receptor was considered severe dysplasia histologically, which might progress to frank malignancy, if not appropriately treated. From this point of view, EGF and its receptor can be clinically regarded as an index of malignant potential of IPMT. On the other hand, EGFR seemed not to be involved in the malignant transformation of MCN. Ten of the 12 MCN, including seven malignant MCN, had no or weak EGFR expression. These data conflict with the series of Kirby et al..$^{20}$ In their series, eight $(61.2 \%)$ of 13 malignant MCN exhibited increased expression of EGFR, whereas EGFR was not detected in any of the 13 benign MCN. This discrepancy needs further clarification. However, the fact that only one of eight malignant MCN had the synchronous expression of EGF and its receptor in our study provided an additional evidence that EGF and its receptor had little role in malignant transformation in MCN. Interestingly, although seven of 10 SPT had a strong expression of EGF, all 10 SPT, including two malignancies, had no or weak EGFR expression. This phenomenon explains the diverse histopathological features and cellular origin, and less malignant potential of SPT compared with other pancreatic cystic neoplasms.

Trefoil factor family (TFF) are abundantly expressed wherever mucin secretion occurs, most predominantly in the mucin secreting cells of the gastrointestinal mucosa. TFF consisted of three trefoil factors (TFF1, TFF2, and TFF3). ${ }^{21}$ It is shown that all TFF peptides are motogens; they stimulate epithelial cell migration in a variety test systems. Of them, TFF1, a single trefoil peptide originally found in breast cancer cell line but produced mainly in the stomach (originally called pS2 or breast cancer oestrogen inducible: BCOI). The 5' flanking region of the TFFl gene contains an enhancer region (EGF/URO) that is responsive to oestrogen, EGF, and other factors. ${ }^{22}$ Rio et al have shown that TFFl could be detected in $67 \%$ of breast tumours that were OR positive and $4 \%$ of carcinomas that were OR negative. ${ }^{23}$ Thus, TFFl might participate a functional oestrogen regulatory system. However, the expression of TFFl can be either oestrogen dependent (as in breast cancer) or oestrogen independent (as in normal gastric epithelium). Welter et al reported a study of TFF 1 expression in human pancreatic cancer. ${ }^{22}$ Of 23 tumours, 17 exhibited significant expression, and the remainder exhibited weak but detectable TFFl immunoreactivity. They concluded that the TFF 1 expression in these tumours was significantly linked to the molecular steps leading to tumourigenesis. Our data supported part of this statement. In the current series, 16 of 20 ductal adenocarcinoma had a strong or moderate expression of TFFl. The high prevalence of TFFl expression in ductal adenocarcinoma in our series might have been induced by an increased expression of EGF and its receptor, instead of by hormonal stimuli. The same phenomenon can be observed in patients with IPMT. The highly increased expression of TFF 1 in IPMT was accompanied by an up-regulation of EGFR in malignant IPMT. In contrast, seven of the $12 \mathrm{MCN}$ had a moderate or strong expression of TFFl that, however, was unrelated to both the hormonal receptors and EGFR. According to our data, TFFl expression seemed not to be directly associated with malignant transformation in MCN. Five of eight malignant MCN and two of four benign MCN had moderate or strong expression of TFFl. These data were in accordance with the series of Kirby et al. ${ }^{20}$ Again, TFFl played no part in SPT. Based on our data, TFFl was not induced in SPT, even though PR was remarkably up-regulated in SPT. Collectively, we speculate that the expression of TFFl could be enhanced by EGFR (as in ductal carcinoma and IPMT), independent of EGFR (as in MCN), and independent of hormonal receptors (as in SPT).

A hormonal influence on the pathogenesis of SPT has been suggested in view of its high prevalence in women. There have been some previous attempts to study the role of OR and PR in SPT, but conflicting results have emerged..$^{1424}$ Nearly all the studies showed no evidence of OR in pancreatic SPT. The only positive findings was that of Landanyi et al, who found OR by biochemical assay, in one woman. ${ }^{25}$ This discrepancy occurred because SPT was reported as immunohistochemically negative for the presence of nuclear OR, whereas a specific binding of ${ }^{3} \mathrm{H}-\mathrm{E} 2$ to cytosol from SPT was performed by Landanyi et al. ${ }^{25}$ The expression of hormonal receptors in stroma beneath the neoplastic epithelium is characteristic for MCN and described as an "ovarian-like stroma." In our study, half of 12 MCN had an expression of hormonal receptors in the corresponding stroma cells but not in the neoplastic epithelium, and all these patients were women. Based on a literature review, in which we found that MCN occurred prevalently in middle aged women, we believe that sex hormone should be deeply involved in the pathogenesis of MCN.

In summary, PR was uniquely expressed in SPT, and OR and PR were expressed in stroma of MCN, reflecting their sex and age predilection. TFFl expression might be related to EGFR such as in IPMT and ductal adenocarcinoma, not related to EGFR such as in MCN, nor related to hormonal receptors such as in SPT. EGF and its receptor might play a part in the malignant transformation of IPMT and ductal adenocarcinoma, but not of SPT and MCN.

\section{Authors' affiliations}

T-S Yeh, Y-Y Jan, K-M Chan, T-L Hwang, M-F Chen, Department of Surgery, Chang Gung Memorial Hospital, Chang Gung University, Taipe

C-T Chiu, Y-B Ho, Department of Gastroenterology, Chang Gung Memorial Hospital

T-C Chen, K-F Lee, Department of Pathology, Chang Gung Memorial Hospital

J-C Hsu, Surgical Laboratory, Chang Gung Memorial Hospital

\section{REFERENCES}

1 Delcore R, Thomas JH, Forster J, et al. Characteristic of cystic neoplasms of the pancreas and results of aggressive surgical treatment. Am J Surg 1992;164:437-42

2 Compagno J, Oertel JE. Mucinous cystic neoplasms of the pancreas with overt and latent malignancy (cystadenocarcinoma and cystadenoma). Am J Clin Pathol 1978;69:573-80.

3 Itai Y, Ohhashi K, Nagai H, et al. "Ductectatic " mucinous cystadenoma and cystadenocarcinoma of the pancreas. Radiology 1986;161:697700

4 Kloppel G, Solcia E, Longnecker DS, et al. Histological typing of tumors of the exocrine pancreas. In: World Health Organization international histological classification of tumours. 2nd edn. Berlin: Springer, 1996:11-20.

5 Tenner S, Carr-Locke DL, Banks PA, et al. Intraductal mucin-hypersecreting neoplasm "mucinous ductal ectasia": endoscopic recognition and management. Am J Gastroenterol 1996:91:2548-54.

6 Rivera JA Castillo F, Pins $M$, et al. Pancreatic mucinous ductal ectasia and intraductal papillary neoplasms. A single malignant clinicopathological entity. Ann Surg 1997;225:6637-46

7 Siech M, Tripp K, Schmidt-Rohlfing B, et al. Intraductal papillary mucinous tumor of the pancreas. Am J Surg 1999;177:117-20.

8 Kobari M, Egawa SI, Shibuya K, et al. Intraductal papillary mucinous tumors of the pancreas comprise 2 clinical subtypes. Arch Surg 1999; 134:1131-6.

9 Nakagohri T, Kenmochi T, Kainuma O, et al. Intraductal papillary mucinous tumors of the pancreas. Am J Surg 1999;178:344-7.

10 Sugiyama $M$, Atomi $Y$, Kuroda A. Two types of mucin-producing cystic tumors of the pancreas: diagnosis and treatment. Surgery 1997; 122:617-25

11 Shy YM, Su CH, Tsay SH, et al. Mucin-producing neoplasms of the pancreas. Ann Surg 1996;223:141-6.

12 Zamboni G, Scarpa A, Bogina G, et al. Mucinous cystic tumors of the pancreas. Am J Surg Pathol 1999;23:410-22.

13 Pettinato G, Manivel C, Ravetto C, et al. Papillary cystic tumor of the pancreas. Am J Clin Pathol 1992;98:478-88.

14 Lam KY, Lo CY, Fan ST. Pancreatic solid-cystic-papillary tumor: clinicopathologic features in eight patients from Hong Kong and review of the literature. World J Surg 1999;23:1045-50. 
15 Jeng LB, Chen MF, Tang RP. Solid and papillary neoplasm of the pancreas. Emphasis on surgical treatment. Arch Surg 1993;128:433-6.

16 Reiner A, Neumeister B, Spona J, et al. Immunohistochemical localization of estrogen and progesterone receptor and prognosis in human primary breast cancer. Cancer Res 1990;50:7057-61.

17 Korc M, Meltzer P, Trent J. Enhanced expression of epidermal growth factor receptor correlates with alterations chromosome 7 in human pancreatic cancer. Proc Nat Acad Sci USA 1986;83:5141-4.

18 Downward J, Yaden Y, Mates E, et al. Close similarity of epidermal growth factor receptor and v-erb-B oncogene protein sequence. Nature 1984;307:521-7.

19 Chen YF, Pan GZ, Hou X, et al. Epidermal growth factor and its receptors in human pancreatic carcinoma. Pancreas 1990;5:278-83.

20 Kirby RE, Lewandrowski KB, Southern JF, et al. Relation of epidermal growth factor receptor and estrogen receptor-independent pS2 protein to the malignant transformation of mucinous cystic neoplasms of the pancreas. Arch Surg 1995; 130:69-72.

21 Wright NA, Hoffmann W, Otto WR, et al. Rolling in the clover: trefoil factor family (TFF)-domain peptides, cell migration and cancer. FEBS Lett 1997;408:121-3

22 Welter C, Theisinger B, Seitz G, et al. Association of the human spasmolytic polypeptide and an estrogen-induced breast cancer protein (pS2) with human pancreatic carcinoma lab Invest 1992:66:187-92. 3 Rio M, Bellock JR, Rasmussen VB, et al. Specific expression of the pS2 gene in subclasses of breast cancers in comparison with expression of the estrogen and progesterone receptors and the oncogene erbB-2. Proc Natl Acad Sci USA 1987;84:9243-7.

24 Carbone A, Ranelletti FO, Rinelli A, et al. Type II estrogen receptor in the papillary cystic tumor of the pancreas. Am J Clin Pathol 1989;92:572-6.

25 Ladanyi M, Mulay S, Arseneau J, et al. Estrogen and progesterone receptor determination in the papillary cystic neoplasm of the pancreas. Cancer 1987;60:1604-11.

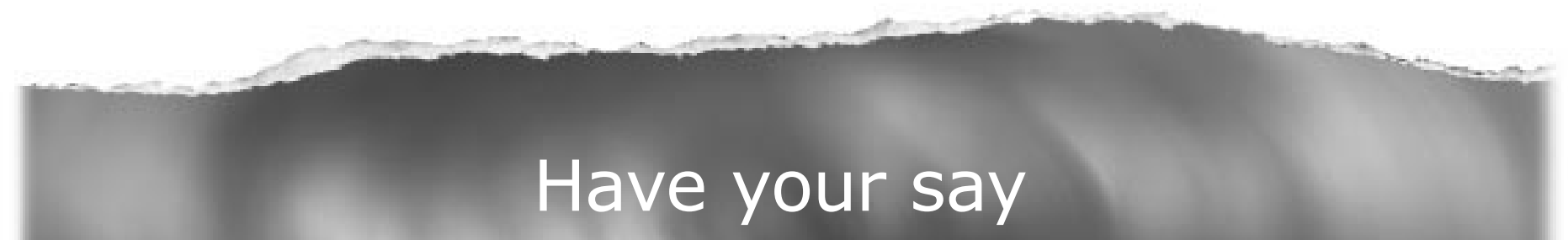

\section{eLetters}

If you wish to comment on any article published in Gut you can send an eletter using the eLetters link at the beginning of each article. Your response will be posted on Gut online within a few days of receipt (subject to editorial screening).

www.gutjnl.com 